\title{
Actin pedestal formation by enteropathogenic Escherichia coli and intracellular motility of Shigella flexneri are abolished in $\mathrm{N}$-WASP-defective cells
}

\author{
Silvia Lommel ${ }^{1,2}$, Stefanie Benesch ${ }^{2}$, Klemens Rottner ${ }^{2}$, Thomas Franz ${ }^{3}$, Jürgen Wehland ${ }^{2,+}$ \\ \& Ralf Kühn
}

${ }^{1}$ Institute for Genetics, University of Köln, Weyertal 121, 50931 Köln, ${ }^{2}$ Department of Cell Biology, Gesellschaft für Biotechnologische Forschung (GBF), Mascheroder Weg 1, 38124 Braunschweig and ${ }^{3}$ Institute of Anatomy, University of Bonn, Nussallee 10, 53115 Bonn, Germany

Received July 9, 2001; revised and accepted August 10, 2001

In mammalian cells, actin dynamics is tightly controlled through small GTPases of the Rho family, WASP/Scar proteins and the Arp2/3 complex. We employed Cre/loxP-mediated gene targeting to disrupt the ubiquitously expressed N-WASP in the mouse germline, which led to embryonic lethality. To elucidate the role of N-WASP at the cellular level, we immortalized embryonic fibroblasts and selected various N-WASPdefective cell lines. These fibroblasts showed no apparent morphological alterations and were highly responsive to the induction of filopodia, but failed to support the motility of Shigella flexneri. In addition, enteropathogenic Escherichia coli were incapable of inducing the formation of actin pedestals in N-WASP-defective cells. Our results prove the essential role of this protein for actin cytoskeletal changes induced by these bacterial pathogens in vivo and in addition show for the first time that N-WASP is dispensible for filopodia formation.

\section{INTRODUCTION}

Actin filament assembly and turnover occurs in response to various extracellular stimuli and drives many cellular activities such as cell locomotion, cellular morphogenesis and phagocytosis. One prerequisite for actin reorganization is the de novo nucleation of actin filaments, which is also stimulated by intracellular pathogens that subvert the actin cytoskeleton and which have, therefore, been instrumental in identifying the essential factors involved in this process (Frischknecht and Way, 2001). Members of the Wiskott-Aldrich Syndrome protein (WASP)/Scar family promote actin polymerization by stimulating the actinnucleating activity of the Arp2/3 complex (reviewed in Takenawa and Miki, 2001). In contrast to Scar proteins, hematopoietic WASP and ubiquitously expressed N-WASP directly interact with the Rho-GTPase Cdc42 through their CRIB (Cdc42/ Rac-interactive-binding) domain (Aspenstrom et al., 1996; Rohatgi et al., 1999). Cdc42 induces the formation of cell surface projections such as filopodia (Kozma et al., 1995; Nobes and Hall, 1995), which is enhanced upon expression of N-WASP (Miki et al., 1998). This suggests that filopodia formation results from Cdc42-based activation of N-WASP, which leads to Arp2/3 recruitment and subsequent actin filament assembly (Carlier et al., 1999). In addition, WASP/N-WASP have been suggested to be involved in the formation of actin pseudopodia by enteropathogenic Escherichia coli (EPEC) (Kalman et al., 1999), whereas intracellular Shigella flexneri and vaccinia virus have been shown to engage N-WASP for initiating actin polymerization at their surfaces in the host cell cytoplasm (Egile et al., 1999; Frischknecht et al., 1999).

In order to analyze N-WASP function in detail, we generated conditional knockout mice and blocked N-WASP expression during embryonic development. By establishing N-WASPdefective fibroblasts we were able to elucidate essential roles of $\mathrm{N}$-WASP function at the cellular level.

+Corresponding author. Tel: +49 5316181 415; Fax: +49 5316181 444; E-mail: jwe@ gbf.de

Present address: Artemis Pharmaceuticals GmbH, Neurather Ring1, 51063 Köln, Germany

$\mathrm{S}$. Benesch and K. Rottner contributed equally to this work 


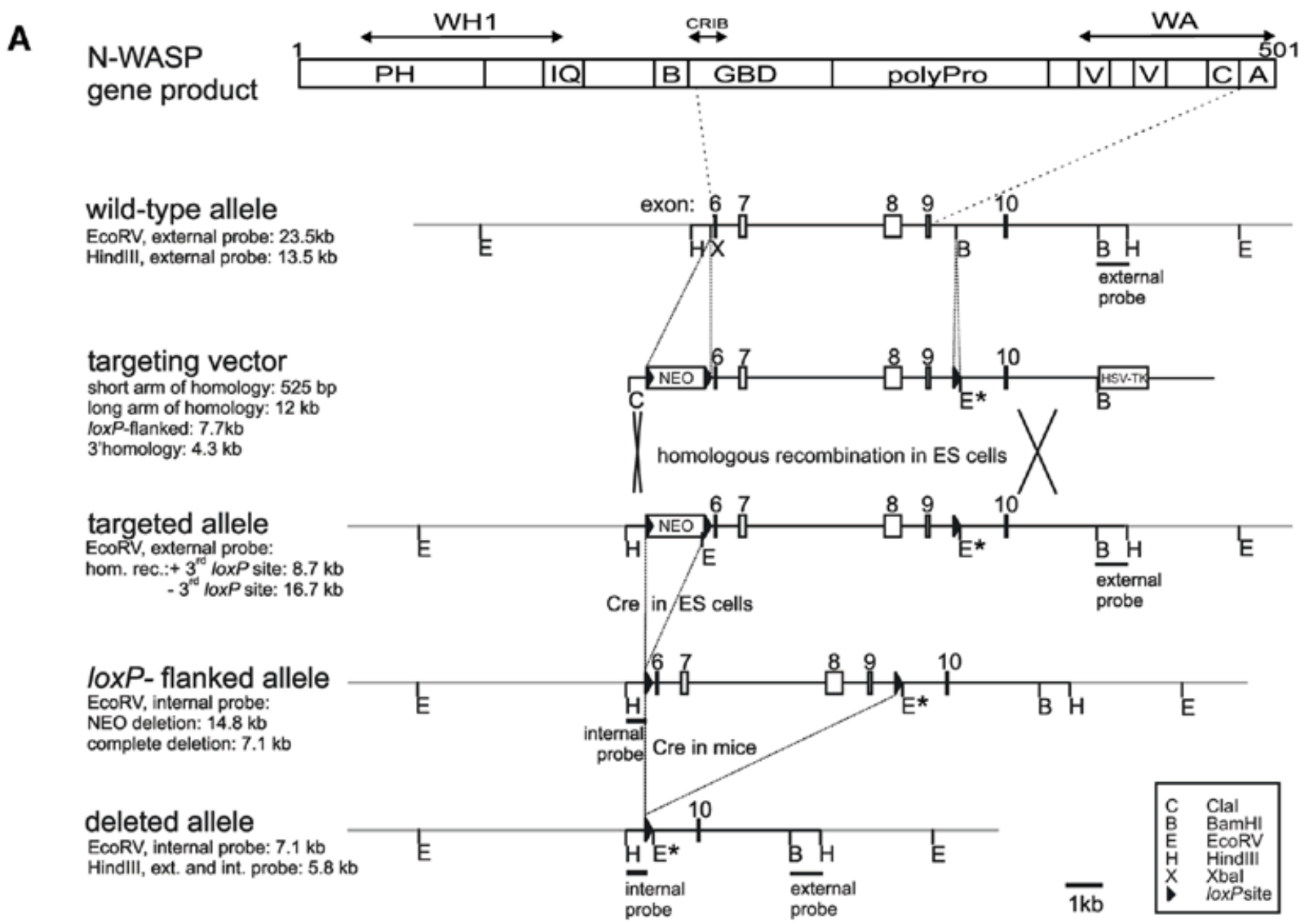

Fig. 1. Disruption of N-WASP leads to embryonic lethality. (A) Schematic representation of the N-WASP protein and the gene targeting strategy showing the genomic organization of the N-WASP locus in wild-type, targeted, floxed and deleted alleles. Also shown are the targeting vector and external and internal probes used for Southern blot analysis. Note the introduction of a new EcoRV restriction site upon gene targeting (marked EcoRV*) used to distinguish the different alleles.

\section{RESULTS AND DISCUSSION}

\section{N-WASP knockout leads to embryonic lethality}

We generated conditionally gene targeted mice on a C57BL/6 background using the phage $\mathrm{P} 1$-derived Cre/loxP recombination system. To inactivate N-WASP we flanked the putative exons 6-9 with IoxP sites by homologous recombination in embryonic stem (ES) cells (Figure 1A-C). After excision of the neo-selection marker in targeted ES cells by transient expression of Cre recombinase (Figure 1C), we derived germline targeted offspring carrying one loxP-flanked allele (N-WASPflox/+) (Figure 1D and E). N-WASPfloxfllox mice behaved and bred normally showing no obvious alteration in N-WASP expression (Figure 1F). Excision of exons $6-9$ by Cre eliminates N-WASP effector domains (Figure $1 \mathrm{~A}$ ) and splicing of exon 5 to the remaining exon 10 creates a premature stop codon (data not shown). After crossing with the transgenic mouse strain Cre159, which restricts Cremediated deletion to neurons in the forebrain (Minichiello et al., 1999), expression of N-WASP protein was strongly reduced in extracts from forebrains of N-WASPfloxflox Cre159 mice as expected (Figure 1F), proving the successful targeting strategy. To assess the physiological consequences of N-WASP deficiency during embryonic development, we disrupted N-WASP in the mouse germline by crossing with a deleter-Cre mouse strain (Schwenk et al., 1995), leading to a constitutive N-WASP knockout (N-WASPdel/del), which turned out to be embryonic lethal. Whereas heterozygous N-WASPde/wt mice appeared normal, no homozygous N-WASPdeldel offspring were born. Thirty-nine implants were histologically analyzed in serial sections on embryonic day (E) 8.5, E9.0 and E9.5 and 79 embryos were dissected on days E9.5 to E13.5. Ten (25.6\%) and 22 (27.8\%) embryos, respectively, revealed developmental abnormalities and/or were partially resorbed. Upon genotyping of the dissected embryos by PCR, developmental abnormalities were found to correspond to the homozygous N-WASPdel/del genotype. Thus, the abnormal embryos detected in the histological analysis shown in Figure 1J (see also Supplementary figure 1) most likely represent N-WASPdel/del embryos. Figure $1 \mathrm{I}$ and J show a comparison of phenotypically normal and N-WASPdel/del embryos demonstrating developmental retardation and reduced growth of N-WASPdel/del embryos, which failed to undergo turning and showed abnormalities of intra- and extra-embryonal mesoderm differentiation (for further details see Supplementary data).

\section{Characterization of N-WASPdel/del cell lines}

Embryonic N-WASPfloxflox fibroblasts were immortalized, subcolned and various N-WASPdel/del cell lines were selected after 


\section{scientific reports}

\section{S. Lommel et al.}
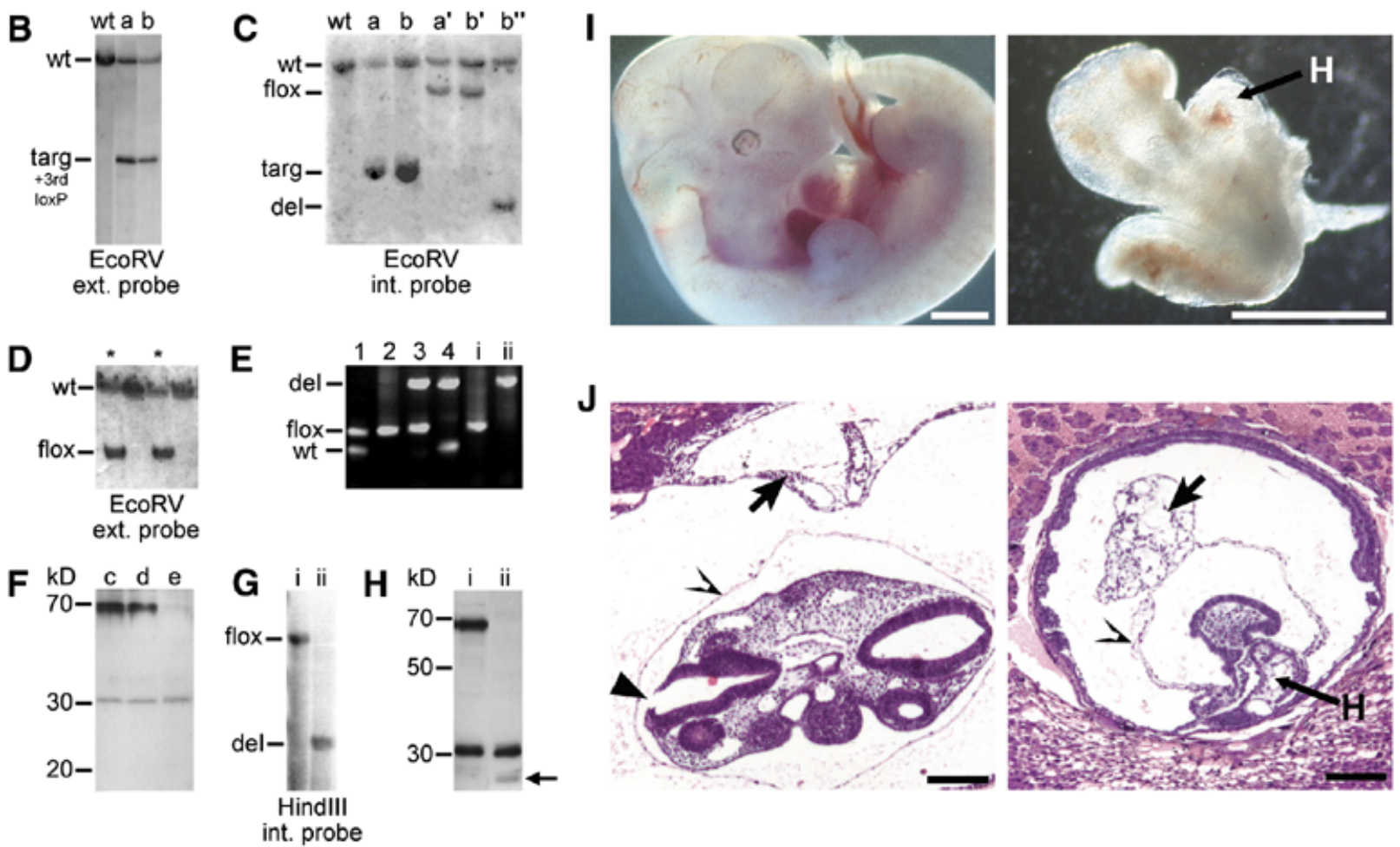

Fig. 1. (continued) (B and C) Southern analyses of precursor (wt), targeted (a and b), floxed (N-WASPlox/wt) (a', $\left.b^{\prime}\right)$, and deleted (N-WASPdel/wt) (b") ES clones (D) Southern analysis of N-WASP flox/wt mice (marked with asterisks) derived after germline transmission. (E) PCR for genotypes of mice (lanes 1-4) and fibroblast cell lines (i and ii): wild-type, floxed and deleted alleles. Note the absence of the N-WASPlox allele in the N-WASPdel/del cell line (ii). (F) Western blot analysis of N-WASP in extracts of hippocampi of wild-type (c), N-WASPflox/flox (d) and N-WASPfloxflox Cre159 mice (e) with polyclonal antibodies against the N-terminus of N-WASP. (G) Southern and (H) Western blot analyses of a representative N-WASPflox/flox fibroblast precursor cell line (i) and its corresponding N-WASPdel/del line (ii). (H) Note the lack of N-WASP full-length expression in (ii). In both (F) and (H), the crossreaction at $\sim 30 \mathrm{kDa}$ was used as loading control. The small polypeptide of $\sim 23 \mathrm{kD}$ detected in extracts of N-WASPdel/del fibroblasts is indicated by an arrow. This product was not detected in forebrain extracts from Cre159 transgenic animals (F, lane e). (I) Comparison of genotyped embryos from intercrosses of N-WASPdel/wt mice at E11.5. Note the reduced size and developmental retardation of the N-WASPdel/del embryo (right) as compared with its wild-type sibling (left), H points to heart. Bars, 1 mm. (J) Histological analyses of presumptive wild-type (left) and N-WASPdel/del embryo (right) at E9 from intercrosses of N-WASPdel/wt mice. The cranial parts of the wild-type embryo are well developed showing pharyngeal arches, a large brain cavity and an almost closed cranial neuropore in the hindbrain area (filled arrowhead). The heavily retarded embryo has not turned, but has formed a heart (marked $\mathrm{H}$ ) and cranial mesenchyme, whereas the allantois (short arrow) shows widening of the intercellular spaces distally and does not contribute to the placenta as in wild-type. Open arrowheads point at amnion. Bars, $150 \mu \mathrm{m}$.

transient expression of Cre, thus enabling a direct comparison with their respective parental precursor lines. Figure $1 \mathrm{G}$ shows a Southern blot and Figure 1E shows PCR analysis of a representative N-WASPdel/del cell line and its N-WASPfloxflox parental precursor line. Western blot analysis demonstrated lack of expression of full-length N-WASP in the N-WASPdel/del cell line (Figure $1 \mathrm{H})$. The weak labelling of a polypeptide with an estimated molecular weight of $23 \mathrm{kDa}$, which was recognized with two independent antibodies against the $\mathrm{N}$-terminus of $\mathrm{N}$-WASP, obviously corresponds to a truncated polypeptide (exons 1-5). However, we could not detect such a truncated gene product in extracts derived from conditionally N-WASP mutant mice (Figure 1F).

\section{Induction of filopodia by $\mathrm{Cdc} 42$ does not require N-WASP}

Rho family GTPases have been recognized as key molecules in the regulation of actin-based cell motility (Hall, 1998), of which Cdc42 is thought to induce the formation of filopodia (Kozma et al., 1995; Nobes and Hall, 1995). Several effector proteins that interact specifically with GTP-Cdc42 have been identified to date (Erickson and Cerione, 2001), but only N-WASP (Miki et al., 1998; Rohatgi et al., 1999) and the hematopoietic WASP (Symons et al., 1996) provide a direct link with actin assembly. The ubiquitously expressed N-WASP was therefore proposed to effect the formation of filopodia (Miki et al., 1998). Interestingly, none of our N-WASP-defective cell lines showed any apparent abnormalities in cell morphology compared with their respective precursor cell lines (not shown). We therefore sought to test whether the formation of filopodia induced by constitutively active Cdc42 is altered in N-WASP-defective cells. Nobes and Hall (1995) microinjected a mixture of constitutively active Cdc42, dominant-negative Rac (to suppress lamellipodia) and C3-transferase (to inhibit Rho), which caused the formation of filopodia in Swiss 3 T3 fibroblasts. Microinjection of the same mixture into precursor (N-WASPfloxflox) and N-WASP-defective cells led to the rapid formation of numerous filopodia (Figure 2A and B, see also Supplementary video 1) in both cell types. Quantitation of filopodia $\sim 30 \mathrm{~min}$ after microinjection revealed no difference between the average number of filopodia induced in N-WASPflox/flox (42 $\pm 13, n=6)$ and in N-WASP-defective cells 


\section{scientific reports}

$N$-WASP and actin dynamics
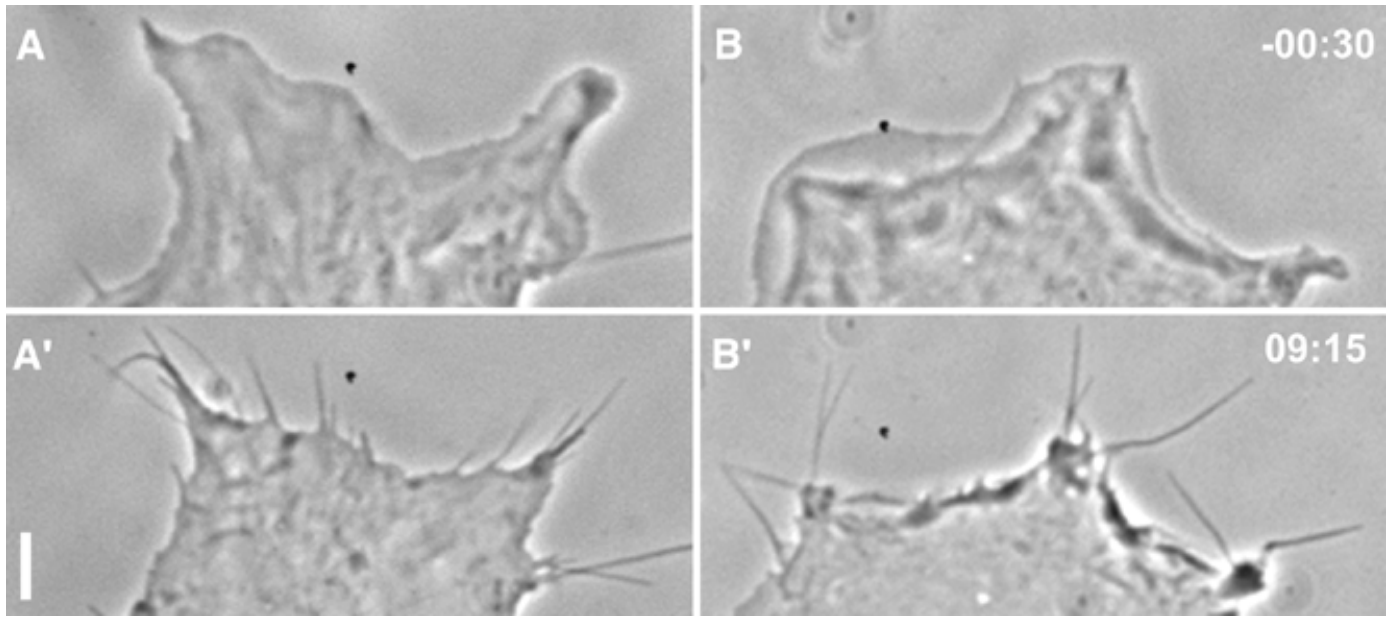

Fig. 2. N-WASP is not required for Cdc42-induced filopodia formation. Cells were microinjected with a mixture of L61Cdc $42(1.5 \mathrm{mg} / \mathrm{ml}), \mathrm{N} 17 \mathrm{Rac}(0.35 \mathrm{mg} / \mathrm{ml})$ and C3-transferase $(0.1 \mathrm{mg} / \mathrm{ml})$. Phase contrast images show a precursor $\left(\mathbf{A}, \mathbf{A}^{\prime}\right)$ and a corresponding N-WASP-defective cell $\left(\mathbf{B}, \mathbf{B}^{\prime}\right)$ before (A, B) and after $\left(\mathrm{A}^{\prime}, \mathrm{B}^{\prime}\right)$ microinjection, which induced the formation of filopodia in both cell types. Approximate times before and after injections are given in minutes and seconds. Bar, $5 \mu \mathrm{m}$.

$(48 \pm 16, n=9)$. Thus, our data demonstrate for the first time that, at least in fibroblasts, N-WASP is not essential for the protrusion of filopodia induced by Cdc42.

\section{Infection of N-WASP-defective cells with intracellular bacterial pathogens}

The bacterial pathogens S. flexneri and Listeria monocytogenes gain access to the host cytoplasm of infected cells where they induce the polymerization of actin filaments at their surfaces, which leads to the formation of comet-like actin tails, a process that provides the driving force for bacterial propulsion. In the case of Shigella, interaction of N-WASP with the bacterial surface protein IcsA/VirG is thought to unfold N-WASP leading to recruitment of the Arp2/3 complex and its activation, whereas the Listeria surface protein ActA recruits and activates the Arp2/3 complex directly (for references see Frischknecht and Way, 2001). In addition, the Rho-GTPase Cdc42 has been suggested to contribute to the actin-based motility of Shigella by initiating actin nucleation through formation of the IcsA/N-WASP/Arp2/3 complex (Suzuki et al., 2000). When N-WASP-defective fibroblasts were infected with $S$. flexneri actin-tail formation was completely abolished (compare Figure $3 \mathrm{C}$ with $\mathrm{B}$ ), whereas tail formation by Listeria was unaffected in these cells (Figure 3A). Shigella-induced actin tail formation could be fully restored upon ectopic expression of full-length murine N-WASP fused to GFP (Figure 3D) as well as by expression of N-WASP-H208D that is incapable of binding to GTP-Cdc42 (Figure 3E) (Miki et al., 1998 and Supplementary figure 3 ) and a $\Delta$ (B-CRIB) mutant (NWASP- $\triangle$ aa160-225) (Figure 3F). In addition, time-lapse movies of Shigella-infected N-WASP-defective cells transfected with full-length N-WASP, N-WASP-H208D (see also Supplementary video 2 ) or with the $\Delta$ (B-CRIB) mutant (not shown) revealed that the incapability of these mutant proteins to interact with Cdc42 (Supplementary figure 3) does not decrease Shigella motility. Since the H208D mutant of N-WASP was shown previously to be recruited to the Shigella surface (Suzuki et al., 2000), it was suggested that IcsA and Cdc42 bind to different motifs in N-WASP
(Frischknecht and Way, 2001). Whereas our data confirm this hypothesis, they clearly demonstrate a recruitment mechanism independent of the CRIB domain. Furthermore, both the N-WASP construct B-GBD (GTPase binding domain) (aa 156-274, see Figure $3 \mathrm{~K}$ ) and the $\mathrm{WH} 1$ domain (aa 1-154) were recruited to the Shigella surface without inducing actin tails (Figure $3 \mathrm{H}$ and $\mathrm{G}$, respectively), which was also observed when N-WASP- $\Delta$ WA was used as a control (Figure 3I). Previously, residues 148-273 of N-WASP were shown to target to the Shigella surface under experimental conditions where one can not exclude an interaction with endogenous N-WASP (Moreau et al., 2000), since residues 209-274 were shown thereafter to interact intramolecularly with the C-terminal cofilin-homology-domain of $\mathrm{N}-\mathrm{WASP}$ in vitro (Prehoda et al., 2000). Our data refine the recruitment domain originally mapped by Moreau et al. (2000) to residues 226-273 within the GBD, but in addition suggest an alternative, WH1-domain-mediated recruitment mechanism, the nature and significance of which is currently unknown. Interestingly, constructs that lack the $\mathrm{WH} 1$ domain such as $\Delta(\mathrm{WH} 1$ CRIB) (Figure 3J) or mini-N-WASP (not shown, see summary in Figure $3 \mathrm{~K}$ ) were still capable of restoring actin tail formation, although average tail lengths appeared to be decreased as compared with full-length N-WASP. Whether this difference reflects a decrease in Shigella motility is currently under investigation. N-WASP lacking the polyproline region was still capable of restoring Shigella-induced actin tail formation, although again with less efficiency (not shown). This may be explained by the lack of direct interaction of the latter mutant with profilin I (Suetsugu et al., 1998), which has previously been shown to play a significant role in Shigella motility (Mimuro et al., 2000). Together, in contrast to previous data (Suzuki et al., 2000), we did not detect any apparent differences between Shigella-induced actin-tail formation upon rescue with full-length N-WASP as compared with $\mathrm{H} 208 \mathrm{D}$ or $\Delta(\mathrm{B}-\mathrm{CRIB})$ mutants. We conclude that Cdc42 is not essential for the actin-driven motility of Shigella. Our conclusions corroborate both the observation that Shigellainduced actin tail formation is not impaired in infected cells treated with the clostridial toxin TcdB-10463, which blocks the 


\section{scientific reports}

\section{S. Lommel et al.}
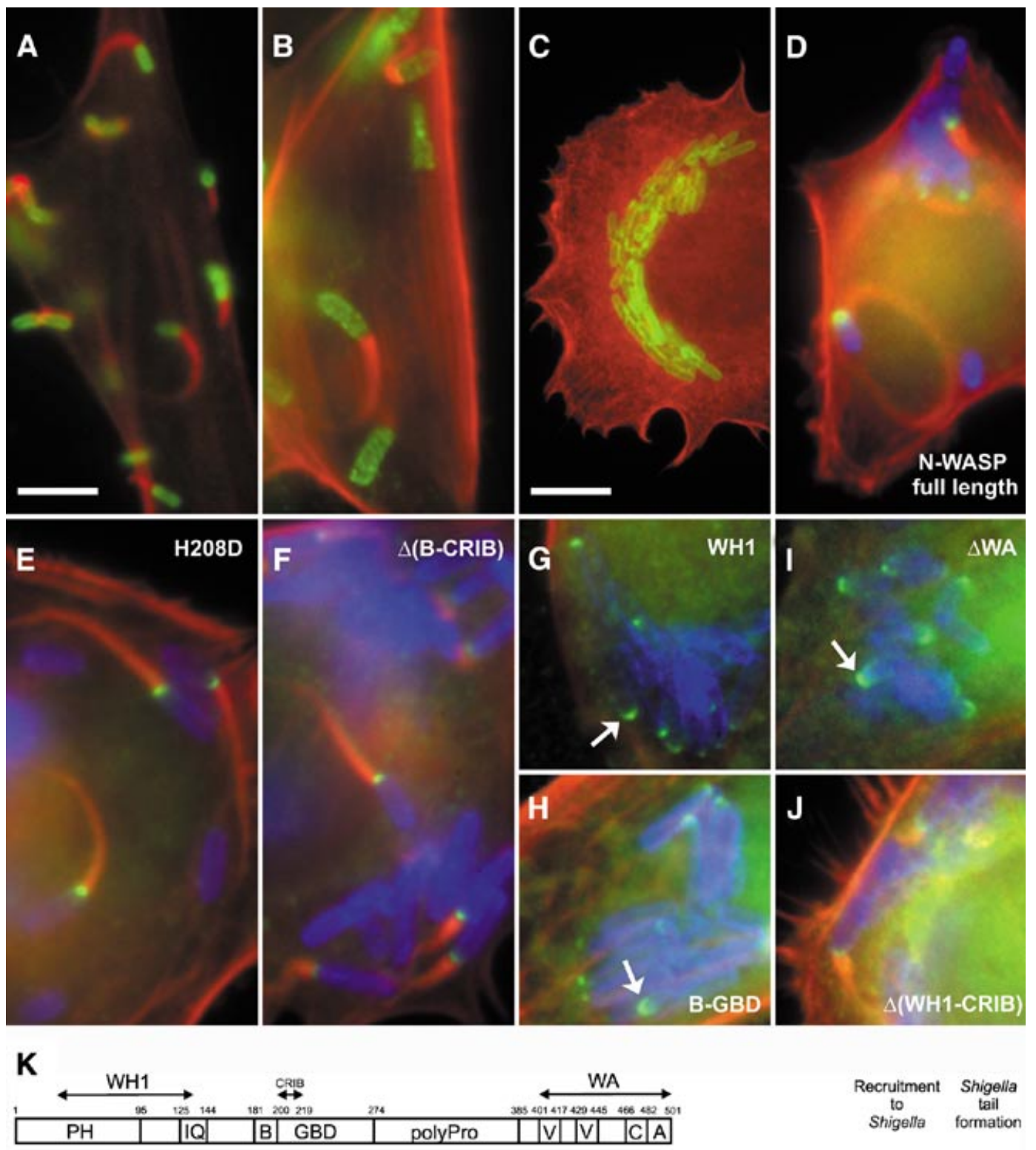

\begin{tabular}{|c|c|c|}
\hline & $\begin{array}{c}\text { Recruitment } \\
\text { to } \\
\text { Shigella }\end{array}$ & $\begin{array}{c}\text { Shigella } \\
\text { tail } \\
\text { formation }\end{array}$ \\
\hline N-WASP full length & + & + \\
\hline WH1 & + & - \\
\hline WH1-GBD & + & - \\
\hline$\triangle W A$ & + & - \\
\hline$\Delta$ (WH1-CRIB) & + & $++^{*}$ \\
\hline WA & - & - \\
\hline B-GBD & + & - \\
\hline $\mathrm{H} 208 \mathrm{D}$ & + & + \\
\hline$\Delta(\mathrm{B}-\mathrm{CRIB})$ & + & + \\
\hline$\Delta$ polyPro & + & + \\
\hline mini-N-WASP & + & $++^{*}$ \\
\hline
\end{tabular}

Fig. 3. N-WASP is essential for Shigella-induced actin tail formation. Precursor (B) and N-WASP-defective fibroblasts (A and C-J) were infected with L. monocytogenes (A) or S. flexneri (B-J). (A-C) show non-transfected cells, while (D-J) show cells expressing GFP-tagged N-WASP constructs as indicated. In all images, filamentous actin is shown in red. Listeria (A) and Shigella (B, C) are shown in green. In (D-J), Shigella and GFP fusion proteins are labeled blue and green, respectively. Listeria form actin tails in N-WASP-defective fibroblasts (A), while Shigella-induced actin tails are abolished in these cells (C), in contrast to the precursor cell line (B). Actin tail formation by Shigella is restored upon expression of full-length N-WASP (D), H208D, $\Delta(\mathrm{B}-\mathrm{CRIB})$ and $\Delta(\mathrm{WH} 1-\mathrm{CRIB})$ mutants (E, F and J, respectively), but not after expression of N-WASP-WH1 (G), -B-GBD (H) or N-WASP- $\Delta$ WA (I), although the three latter constructs are recruited to the Shigella surface (indicated by arrows). Bar in (A) $(5 \mu \mathrm{m})$ is valid for (A-J) except $(\mathrm{C})(10 \mu \mathrm{m})$. (K) shows the domain structure of N-WASP and an overview of the GFP-tagged constructs used in this study. Recruitment to Shigella and reconstitution of actin tail formation are given. +* marks low efficiency and/or shorter tails; PH, pleckstrin-homology domain; IQ, calmodulin-binding motif; B, basic domain; GBD, GTPase binding domain; polyPro, polyproline region; $\mathrm{V}$, verprolin homology domain; $\mathrm{C}$, cofilin homology domain and $\mathrm{A}$, acidic domain. 


\section{scientific reports}

GTPases of the Rho family including Cdc42 (Mounier et al., 1999), and the fact that, in a reconstituted cell-free system, Cdc42 is not required for Shigella-motility (Loisel et al., 1999).

\section{Enteropathogenic E. coli (EPEC)-induced formation of actin pedestals requires N-WASP}

EPEC subvert the actin cytoskeleton by inducing the formation of characteristic pseudopodial structures (pedestals) with the bacteria residing at their tips (Goosney et al., 2000). Overexpression of a WASP mutant lacking the C-terminal cofilinhomology region has been shown to interfere with pedestal formation in HeLa cells, an effect that was dependent on the CRIB domain of WASP (Kalman et al., 1999). N-WASP, which localizes to the pedestal tips (Goosney et al., 2001; this study), is therefore thought to be required for recruitment and activation of the Arp2/3 complex. It was further proposed that recruitment of WASP/N-WASP involves the Cdc42 homolog Chp (Aronheim et al., 1998; Kalman et al., 1999). When N-WASP-defective fibroblasts were infected with EPEC we found that, although the bacteria readily attached to the cell surface (Figure 4C), pedestal formation was completely blocked (Figure 4D) in contrast to infected precursor cells (Figure 4A and B). Pedestal formation could be fully restored in N-WASP-defective cells by expression of full-length N-WASP (Figure 4E), N-WASP-H208D (Figure 4F) or the $\triangle(B-C R I B)$ mutant (Figure $4 \mathrm{G}$ ), but not by expression of $\mathrm{N}$-WASP mutants truncated at the $\mathrm{N}$-terminus or lacking the WA-effector-domain (summarized in Figure 4K). Furthermore, the WH1-GBD construct (aa 1-274) clearly targeted to the host cell surface (Figure 4I) at sites of EPEC attachment (Figure $4 \mathrm{H}$ ) without inducing formation of actin pedestals (Figure 4J). Thus, the recruitment domain of N-WASP is restricted to its $\mathrm{N}$-terminal half. A construct lacking the polyproline region ( $\Delta$ polyproline, see Figures $3 \mathrm{~K}$ and $4 \mathrm{~K}$ ) also restored pedestal formation, suggesting that a direct interaction of N-WASP with profilin I or $\mathrm{SH} 3$-domain containing adaptor proteins such as Grb2 (Carlier et al., 2000), shown previously to be recruited to EPEC pedestals (Goosney et al., 2001), is not essential for this process. Mini-NWASP, an N-terminally truncated version of the latter construct (see Figure $3 \mathrm{~K}$ and $4 \mathrm{~K}$ ), which corresponds to a mutant that could still be induced by $\mathrm{PIP}_{2}$ (phosphatidylinositol-4-phosphate) and Cdc42 to activate the Arp2/3 complex in vitro (Prehoda et al., 2000), did not restore pedestal formation. Therefore we are currently investigating the role of the $\mathrm{WH} 1$ domain in N-WASP targeting to the attachment site of EPEC, although so far it has been difficult to detect a recruitment of the $\mathrm{WH} 1$ domain alone (not shown). In summary, in contrast to previous results (Kalman et al., 1999), this study clearly shows that recruitment of N-WASP to attachment sites of EPEC at the host cell membrane is not mediated by the CRIB-domain.

\section{Speculation}

$\mathrm{N}$-WASP is a highly potent activator of the Arp2/3 complex in vitro and harbors key regulatory functions for cellular actin assembly. Our study proves the essential role of N-WASP, since disruption of the gene leads to embryonic lethality. In addition, two out of three bacterial pathogens investigated in this study have selected N-WASP as the target molecule to subvert the host cell actin polymerization machinery. This may be due to the presence of an additional verprolin homology region in the C-terminus of N-WASP, which has been correlated with the higher potency of N-WASP to activate the Arp2/3 complex compared with the highly related Scar/WASP family members (Yamaguchi et al., 2000). However, the precise in vivo functions of N-WASP remain to be determined, since it is not clear whether the early embryonic lethality is due solely to disturbances in actin dynamics or may be caused by additional, so far unknown, activities of N-WASP. Nevertheless, the availability of conditionally N-WASP-targeted mice not only enables the generation of specific cell lines for analyzing its role in actinbased motility as described above, but is also a prerequisite for elucidating the physiological functions of N-WASP in vivo by alteration of its temporal and tissue-specific expression.

\section{METHODS}

Transgenic animals. Conditional gene targeting and generation of transgenic animals were performed according to standard protocols and as described in the Supplementary material.

Cell culture and media. Primary embryonic fibroblasts from N-WASPfloxflox mice were prepared on E14 cells and immortalized with retroviruses causing expression of a temperaturesensitive simian virus 40 large-T-antigen (Jat and Sharp, 1989). Fibroblasts were grown at $32{ }^{\circ} \mathrm{C}$ in DMEM with glutamine and $10 \%$ FCS (Life Technologies). Transfections were performed with FuGENE6 (Roche).

N-WASP expression constructs. We isolated the murine N-WASP CDNA by performing independent RT-PCR on RNA from brains of $\mathrm{C} 57 \mathrm{BL} / 6$ mice using primers 5'-AGAGTGGGACCCGAGTGCTC-3' and 5'-ACAGATCAATAGACAGGAAG-3'. For GFPtagged N-WASP expression constructs, murine N-WASP cDNA regions were cloned into $\mathrm{PEGFP-C1}$ or -C2 vectors (Clontech), sequenced and expression was verified by Western blotting (see Supplementary figure 2).

Bacterial strains and infections. We used EPEC strain 2348/69, M90T wild-type invasive strain of $S$. flexneri serotype 5 and L. monocytogenes EGD serotype 1/2a. For EPEC infections, overnight cultures were diluted 1:50 into DMEM and grown for $3 \mathrm{~h}$ at $37^{\circ} \mathrm{C}$. After diluting 1:100, bacteria were centrifuged onto fibroblasts at $700 \mathrm{~g}$ for $5 \mathrm{~min}$ and incubated for $4-5 \mathrm{~h}$ with hourly medium changes. Prior to infections with $S$. flexneri, bacteria grown overnight in trypticase soya bouillion (TSB) were diluted 1:100 into DMEM and centrifuged onto cells. One and a half hours after infection, DMEM was exchanged for DMEM with $10 \%$ FCS and $50 \mu \mathrm{g} / \mathrm{ml}$ gentamicin (Sigma-Aldrich) and incubated for 2 h. Listeria infections were performed as described (Carl et al., 1999).

Immunofluorescence. Indirect immunofluorescence was performed essentially as described (Herzog et al., 1994). We used polyclonal antibodies against $S$. flexneri, monoclonal antibodies against EspE, both provided by $\mathrm{T}$. Chakraborty (Giessen, Germany), polyclonal antibodies against L. monocytogenes (Domann et al., 1992). Secondary reagents were Alexa-Fluor 488-conjugated goat anti-rabbit (Molecular Probes) and AMCAconjugated goat anti-mouse antibodies (Dianova), which were mixed with Alexa 594-phalloidin (Molecular Probes) to stain filamentous actin.

Microinjection, video microscopy and image processing. Recombinant L61-Cdc42, N17Rac and C3-transferase were 


\section{scientific reports}

S. Lommel et al.
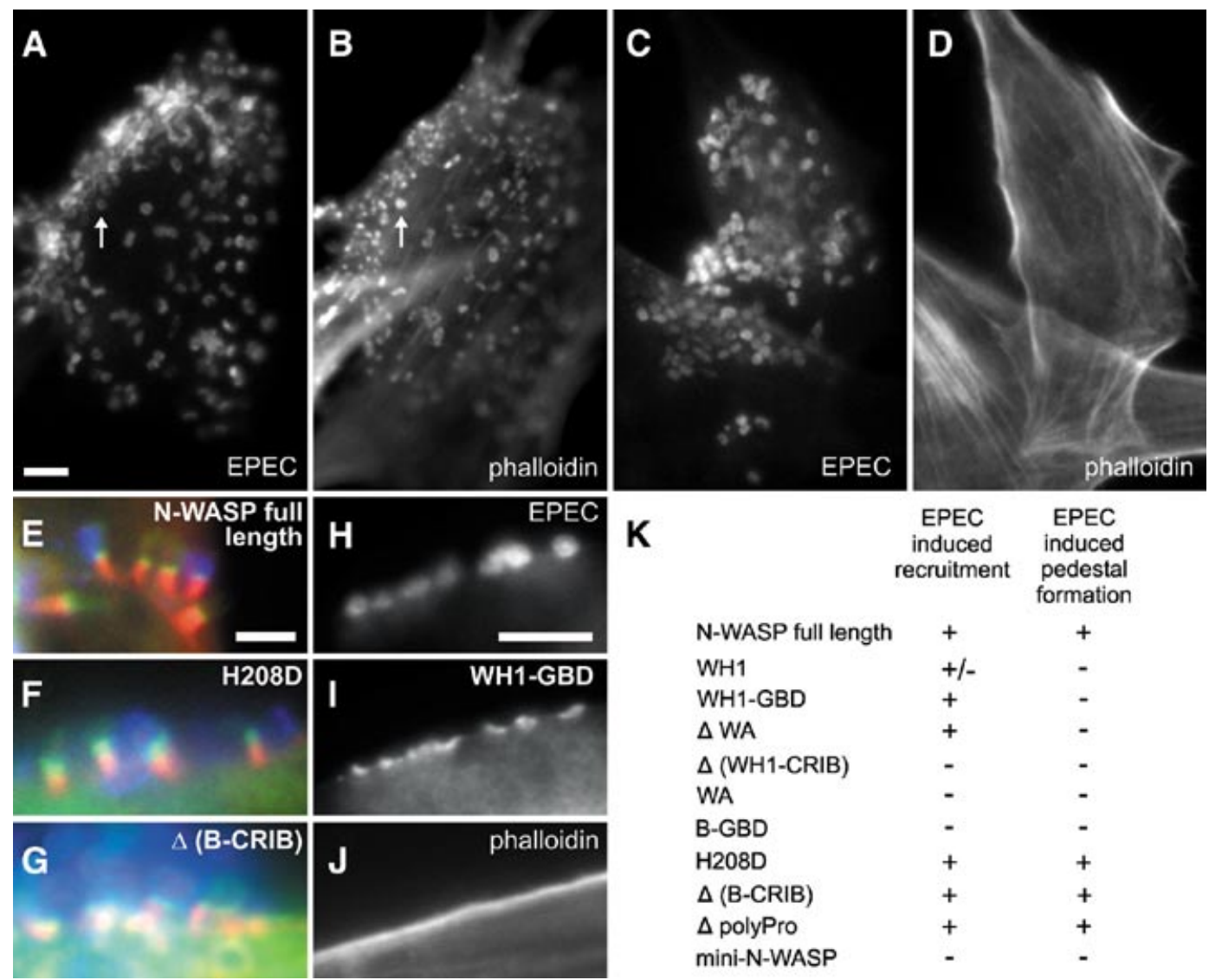

N-WASP full length
WH1
WH1-GBD
$\Delta W A$
$\Delta($ WH1-CRIB)
WA
B-GBD
H208D
$\Delta$ (B-CRIB)
$\Delta$ polyPro
mini-N-WASP

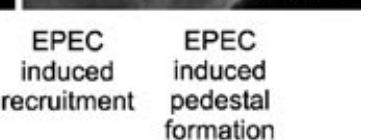

$\begin{array}{cc} & \text { formation } \\ + & + \\ +/- & - \\ + & - \\ + & - \\ - & - \\ - & - \\ - & - \\ + & + \\ + & + \\ + & + \\ - & -\end{array}$

Fig. 4. EPEC pedestal formation depends on N-WASP. Infection of precursor $(\mathbf{A}, \mathbf{B})$ and N-WASP-defective cells $(\mathbf{C}-\mathbf{J})$ with EPEC. EPEC are shown in (A, C and $\mathrm{H}$ ) and in blue in (E-G). F-actin is shown in (B, D and J) and in red in (E-G). GFP constructs are shown in (I) and in green in (E-G). EPEC (A, C)-induced formation of actin pedestals in precursor (B), but not in N-WASP-defective cells (D). Pedestal formation in N-WASP-defective cells was restored upon expression of GFP-tagged full-length N-WASP (E), N-WASP-H208D (F) and N-WASP- $\Delta$ (B-CRIB) (G), while N-WASP-WH1-GBD was recruited (I) by EPEC (H) without induction of actin pedestals $(\mathrm{J})$. Bars $(5 \mu \mathrm{m})$ in $(\mathrm{A}, \mathrm{E}$ and $\mathrm{H})$ are valid for $(\mathrm{A}-\mathrm{D}),(\mathrm{E}-\mathrm{G})$ and $(\mathrm{H}-\mathrm{J})$, respectively. For description of GFP fusion constructs see Figure 3K. (K) The recruitment to EPEC attachment sites and reconstitution of pedestal formation by the respective mutants are indicated.

purified and injected in a mixture essentially as described previously (Nobes and Hall, 1995). Time-lapse movies and fluorescence images were acquired and processed as described (Rottner et al., 1999).

Accession numbers. The murine N-WASP cDNA sequence has been submitted to the DDBJ/EMBL/GenBank Nucleotide Sequence Database under accession No. AJ318416.

Supplementary data. Supplementary data are available at $E M B O$ reports Online.

\section{ACKNOWLEDGEMENTS}

We thank H. Miki for rat N-WASP cDNA used to isolate the murine genomic clone, P. Sharp for the immortalization virus, A. Hall for GTPase constructs, T. Chakraborty for EPEC, antibodies and discussions, A. Steffen for antibodies, K. Rajewsky and W. Müller for generous support, Dave Monner for cell sorting, Theresia Stradal for help with pulldown assays, M. Bernzen, A. Rode, S. Graf, G. Eversloh and M. Klockzin for technical assistance. S.L. was supported by the Volkswagen Foundation (to R.K.) and Artemis Pharmaceuticals GmbH, K.R. by an EMBO long-term fellowship and J.W. by the Deutsche Forschungsgemeinschaft and the Fonds der Chemischen Industrie.

\section{REFERENCES}

Aronheim, A., Broder, Y.C., Cohen, A., Fritsch, A., Belisle, B. and Abo, A. (1998) Chp, a homologue of the GTPase Cdc42Hs, activates the JNK pathway and is implicated in reorganizing the actin cytoskeleton. Curr. Biol., 8, 1125-1128.

Aspenstrom, P., Lindberg, U. and Hall, A. (1996) Two GTPases, Cdc42 and Rac, bind directly to a protein implicated in the immunodeficiency disorder Wiskott-Aldrich syndrome. Curr. Biol., 6, 70-75.

Carl, U.D., Pollmann, M., Orr, E., Gertler, F.B., Chakraborty, T. and Wehland, J. (1999) Aromatic and basic residues within the EVH1 domain of VASP specify its interaction with proline-rich ligands. Curr. Biol., 9, 715-718.

Carlier, M.F., Ducruix, A. and Pantaloni, D. (1999) Signalling to actin: the Cdc42-N-WASP-Arp2/3 connection. Chem. Biol., 6, R235-R240.

Carlier, M.F. et al. (2000) GRB2 links signaling to actin assembly by enhancing interaction of neural Wiskott-Aldrich syndrome protein (N-WASp) with actin-related protein (ARP2/3) complex. J. Biol. Chem., 275, 21946-21952.

Domann, E., Wehland, J., Rohde, M., Pistor, S., Hartl, M., Goebel, W., Leimeister-Wachter, M., Wuenscher, M. and Chakraborty, T. (1992) A novel bacterial virulence gene in Listeria monocytogenes required for host cell microfilament interaction with homology to the proline-rich region of vinculin. ЕМВO J., 11, 1981-1990. 


\section{scientific reports}

Egile, C., Loisel, T.P., Laurent, V., Li, R., Pantaloni, D., Sansonetti, P.J. and Carlier, M.F. (1999) Activation of the CDC42 effector N-WASP by the Shigella flexneri IcsA protein promotes actin nucleation by Arp2/3 complex and bacterial actin-based motility. J. Cell Biol., 146, 1319-1332.

Erickson, J.W. and Cerione, R.A. (2001) Multiple roles for Cdc42 in cell regulation. Curr. Opin. Cell. Biol., 13, 153-157.

Frischknecht, F. and Way, M. (2001) Surfing pathogens and the lessons learned for actin polymerization. Trends Cell Biol., 11, 30-38.

Frischknecht, F., Moreau, V., Rottger, S., Gonfloni, S., Reckmann, I., Superti-Furga, G. and Way, M. (1999) Actin-based motility of vaccinia virus mimics receptor tyrosine kinase signalling. Nature, 401, 926-929.

Goosney, D.L., Gruenheid, S. and Finlay, B.B. (2000) Gut feelings: enteropathogenic E.coli (EPEC) interactions with the host. Annu. Rev. Cell. Dev. Biol., 16, 173-189.

Goosney, D.L., DeVinney, R. and Finlay, B.B. (2001) Recruitment of cytoskeletal and signaling proteins to enteropathogenic and enterohemorrhagic Escherichia coli pedestals. Infect. Immun., 69, 3315-3322.

Hall, A. (1998) Rho GTPases and the actin cytoskeleton. Science, 279, 509-514

Herzog, M., Draeger, A., Ehler, E. and Small, J.V. (1994) Immunofluorescence microscopy of the cytoskeleton: Double and triple immunofluorescence. In Celis, J.E. (ed.), Cell Biology: A Laboratory Handbook. Academic Press, San Diego, CA, pp. 355-360.

Jat, P.S. and Sharp, P.A. (1989) Cell lines established by a temperaturesensitive simian virus 40 large-T-antigen gene are growth restricted at the non-permissive temperature. Mol. Cell. Biol., 9, 1672-1681.

Kalman, D., Weiner, O.D., Goosney, D.L., Sedat, J.W., Finlay, B.B., Abo, A. and Bishop, J.M. (1999) Enteropathogenic E.coli acts through WASP and Arp2/3 complex to form actin pedestals. Nature Cell Biol., 1, 389-391.

Kozma, R., Ahmed, S., Best, A. and Lim, L. (1995) The Ras-related protein $\mathrm{Cdc} 42 \mathrm{Hs}$ and bradykinin promote formation of peripheral actin microspikes and filopodia in Swiss 3 T3 fibroblasts. Mol. Cell. Biol., 15, 1942-1952.

Loisel, T.P., Boujemaa, R., Pantaloni, D. and Carlier, M.F. (1999) Reconstitution of actin-based motility of Listeria and Shigella using pure proteins. Nature, 401, 613-616.

Miki, H., Sasaki, T., Takai, Y. and Takenawa, T. (1998) Induction of filopodium formation by a WASP-related actin-depolymerizing protein N-WASP. Nature, 391, 93-96.

Mimuro, H., Suzuki, T., Suetsugu, S., Miki, H., Takenawa, T. and Sasakawa, C. (2000) Profilin is required for sustaining efficient intra- and intercellular spreading of Shigella flexneri. J. Biol. Chem., 275, 28893-28901.

Minichiello, L. et al. (1999) Essential role for TrkB receptors in hippocampus-mediated learning. Neuron, 24, 401-414.
Moreau, V., Frischknecht, F., Reckmann, I., Vincentelli, R., Rabut, G., Stewart, D. and Way, M. (2000) A complex of N-WASP and WIP integrates signalling cascades that lead to actin polymerization. Nature Cell Biol., 2, 441-448.

Mounier, J., Laurent, V., Hall, A., Fort, P., Carlier, M.F., Sansonetti, P.J. and Coumaran, E. (1999) Rho family GTPases control entry of Shigella flexneri into epithelial cells but not intracellular motility. J. Cell Sci., 112, 2069-2080.

Nobes, C.D. and Hall, A. (1995) Rho, rac, and cdc42 GTPases regulate the assembly of multimolecular focal complexes associated with actin stress fibers, lamellipodia, and filopodia. Cell, 81, 53-62.

Prehoda, K.E., Scott, J.A., Dyche Mullins, R. and Lim, W.A. (2000) Integration of multiple signals through cooperative regulation of the N-WASP-Arp2/3 complex. Science, 290, 801-806.

Rohatgi, R., Ma, L., Miki, H., Lopez, M., Kirchhausen, T., Takenawa, T. and Kirschner, M.W. (1999) The interaction between N-WASP and the Arp2/3 complex links Cdc42-dependent signals to actin assembly. Cell, 97, 221-231.

Rottner, K., Hall, A. and Small, J.V. (1999) Interplay between Rac and Rho in the control of substrate contact dynamics. Curr. Biol., 9, 640-648.

Schwenk, F., Baron, U. and Rajewsky, K. (1995) A cre-transgenic mouse strain for the ubiquitous deletion of loxP-flanked gene segments including deletion in germ cells. Nucleic Acids Res., 23, 5080-5081.

Suetsugu, S., Miki, H. and Takenawa, T. (1998) The essential role of profilin in the assembly of actin for microspike formation. EMBO J., 17, 6516-6526.

Suzuki, T., Mimuro, H., Miki, H., Takenawa, T., Sasaki, T., Nakanishi, H., Takai, Y. and Sasakawa, C. (2000) Rho family GTPase Cdc42 is essential for the actin-based motility of Shigella in mammalian cells. J. Exp. Med., 191, 1905-1920.

Symons, M., Derry, J.M., Karlak, B., Jiang, S., Lemahieu, V., McCormick, F., Francke, U. and Abo, A. (1996) Wiskott-Aldrich syndrome protein, a novel effector for the GTPase CDC42Hs, is implicated in actin polymerization. Cell, 84, 723-734.

Takenawa, T. and Miki, H. (2001) WASP and WAVE family proteins: key molecules for rapid rearrangement of cortical actin filaments and cell movement. J. Cell Sci., 114, 1801-1809.

Yamaguchi, H., Miki, H., Suetsugu, S., Ma, L., Kirschner, M.W. and Takenawa, T. (2000) Two tandem verprolin homology domains are necessary for a strong activation of Arp2/3 complex-induced actin polymerization and induction of microspike formation by N-WASP. Proc. Natl Acad. Sci. USA, 97, 12631-12636.

DOI: 10.1093/embo-reports/kve197 